\title{
Segregation-Induced Degradation in Hydrogen Storage Mg-based Thin Films
}

\author{
Zhi-Peng LI, Shiyou Zheng, Leonid A. Bendersky
}

Materials Science and Engineering Division, National Institute of Standards and Technology, Gaithersburg, Maryland 20899, USA.

One of the criteria in development of efficient hydrogen storage materials is retaining of sorption kinetics during cycling. Degradation of hydrogen sorption is related to microstructural evolution or formation of new phases. There was debate about the possible interaction between hydrogen storage material (i.e. $\mathrm{Mg}$ ) and transition metal (i.e. V, Fe) [1-2]; it is unclear whether Mg-based intermetallic compounds would form and affect changes during hydrogenation. Also, better understanding of the hydrogen migration pathways in Mg-metal based materials is desired. To clarify these points, various techniques of analytic transmission electron microscopy (TEM) have been employed in this work to study hydrogenated states of $\mathrm{Mg}-\mathrm{V}$ and $\mathrm{Mg}$-Fe thin films. The insight into the evolution of morphology and microstructures during hydrogenation and dehydrogenation processes was elucidated, with possible implications for further development of high performance hydrogen storage materials.

In this study, the hydrogen sorption performance tests (data not shown here) of $\mathrm{Mg}_{100-\mathrm{x}} \mathrm{V}_{\mathrm{x}}$ films demonstrated that for $\mathrm{x}>10$ compositions the hydrogen sorption capabilities degrade after cycling processes. In Fig. 1 demonstrates the morphology comparison of $\mathrm{Mg}_{90} \mathrm{~V}_{10}$ films before and after 10 cycling; the HAADF images show (a) a continuous network of a bcc-V phase percolation a Mg matrix for the as-deposited film and (b) formation of sphere-like nanoparticles in the hydrogenated film. The size of segregated nanoparticles increases as increasing number of cycles. The nature of the spherical nanoparticles was determined from high-resolution TEM (HRTEM, Fig. 1c), diffraction and energy dispersive X-ray spectroscopy (EDX) analyses at scanning TEM (STEM) mode (Figs. 1e-h). It was concluded that the nanoparticles are bcc- $\mathrm{V}$ metal. Similar microstructural evolution due to hydrogenation process can be also observed for Mg-Fe thin films (Fig. 2). TEM analysis confirmed that segregated sphere-like nanoparticles are bcc-Fe metal and not metal hydride.

Based on the TEM observations we put forward the following scenario of microstructural evolution during hydrogenation for both $\mathrm{Mg}-\mathrm{V}$ and Mg-Fe films (Fig. 3). Co-depositing of transition metal (TM, i.e. $\mathrm{V}$ or $\mathrm{Fe}$ ) immiscible with $\mathrm{Mg}$ produces a network of continuous layers of TM, which are percolating the Mg matrix; the layers consist of nanograins of either bcc-V or bcc-Fe and divide $\mathrm{Mg}$ into small grains. Such nanocrystalline grains of TM layers act as channels or networks for hydrogenation process, where fast delivery of hydrogen by diffusion of $\mathrm{H}$ atoms in TM would occur. After the hydrogenation cycling, the continuity of the TM layers is disrupted and lead to the aggregation of $\mathrm{V}$ or Fe along the $\mathrm{Mg} / \mathrm{MgH}_{2}$ boundaries into spherical nanoparticles. Whereas the aggregation is driven by Rayleigh morphological instability, hydrogenation process introduces structural defects sufficient to enhance lowtemperature diffusion of TMs to realise the morphological changes. Such microstructural changes interrupt the fast hydrogenation pathway connected by initial V or Fe networks and cause degradation of sorption behaviour. This study thereby provides direct experimental evidence to elucidate enhanced kinetics and degradation issues of the hydrogenation/dehydrogenation processes in Mg-TM films.

References

[1] Tan Z, Chiu C, Heilweil EJ, Bendersky LA, International Journal of Hydrogen Energy 36 (2011) 9702.

[2] Sakintuna B, Lamari DF, Hirscher M, International Journal of Hydrogen Energy 32, (2007) 1121. 

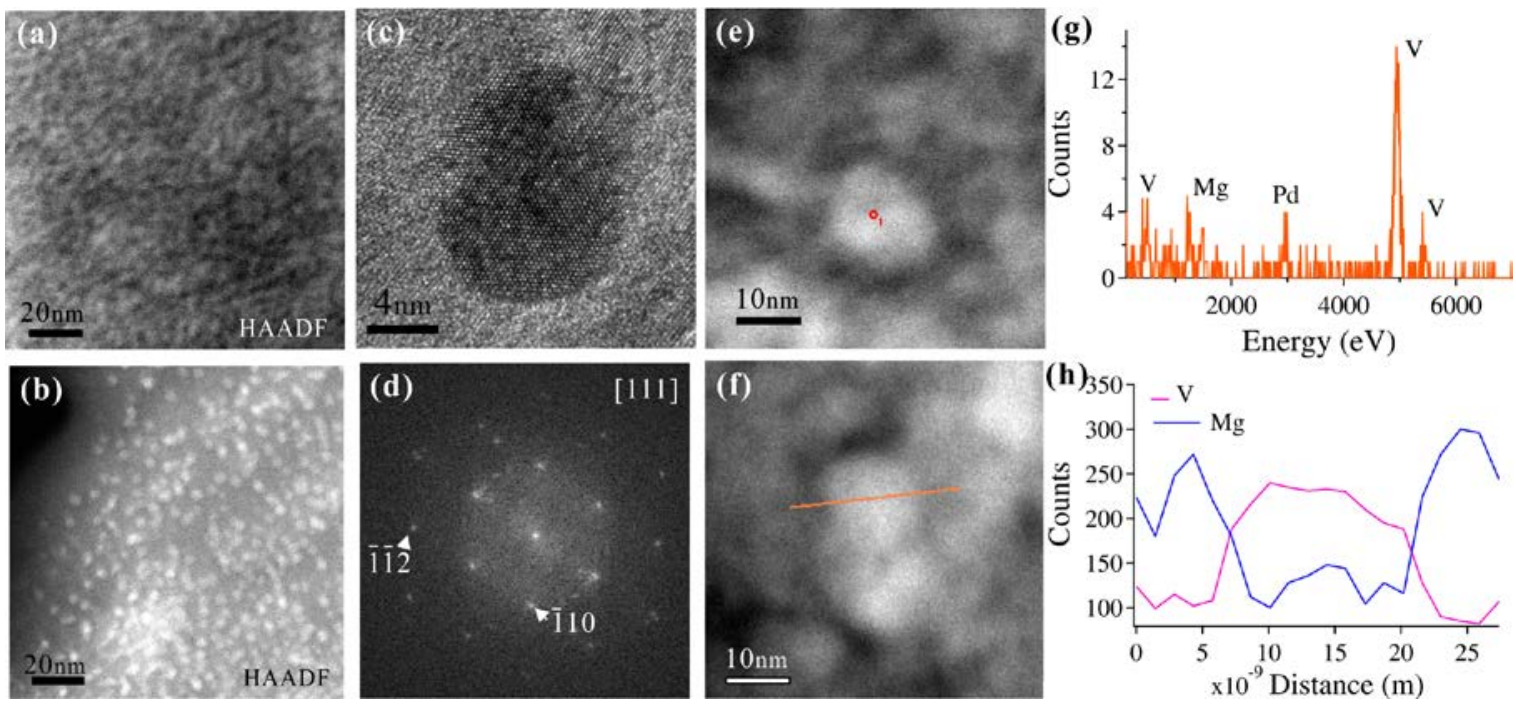

Figure 1. HAADF images of $\mathrm{Mg}_{90} \mathrm{~V}_{10}$ thin film (a) before and (b) after 10 cycles of $\mathrm{H}_{2}$ sorption processes. (c) HRTEM observation of sphere-like nanoparticle appeared in (b), the corresponding FFT result is shown in (d). The STEM EDX point scan and line scan results are shown in $(\mathrm{e}) \&(\mathrm{~g})$ and (f)\&(h), respectively.

Figure 2
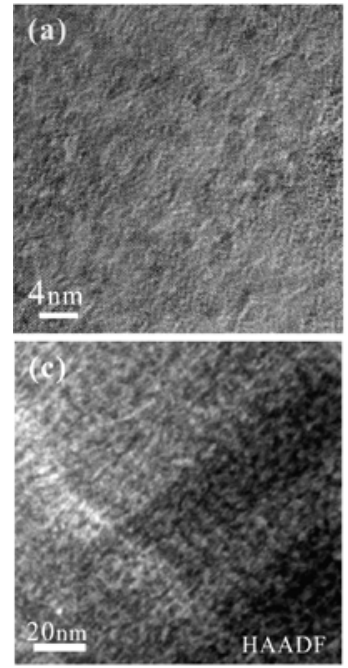

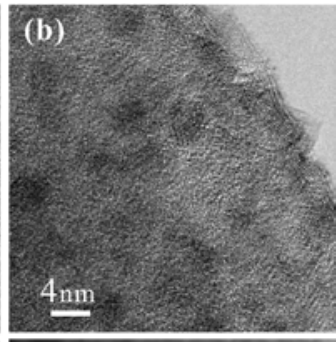

(d)

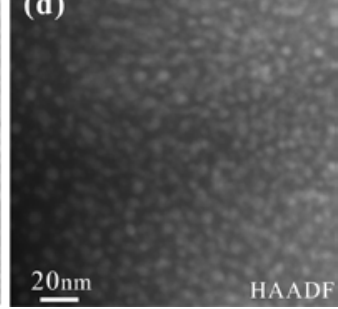

Figure 3

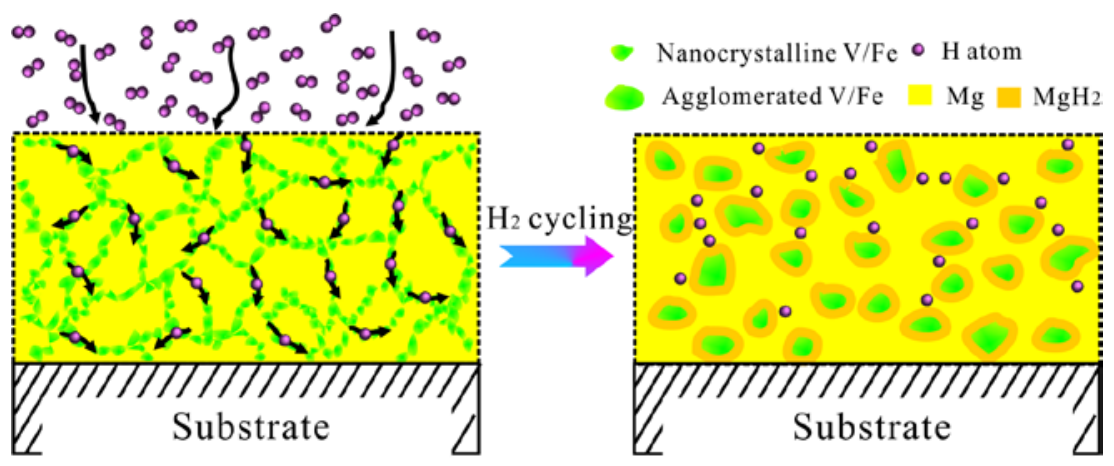

Figure 2. HRTEM and HAADF images of $\mathrm{Mg}_{85} \mathrm{Fe}_{15}$ thin film without $\mathrm{H}_{2}$ treatment (a,c) and after 10 cycles of hydrogenation processes $(b, d)$.

Figure 3. Schematic diagram of hydrogen induced transition metal (i.e. V or Fe) segregation in $\mathrm{Mg}$ thin films, and related mechanism for the degradation of hydrogenation process due to such segregation of transition metals in thin film. 